\title{
Lipid-lowering and anti-inflammatory effects of omega 3 ethyl esters and krill oil: a randomized, cross-over, clinical trial
}

\author{
Arrigo F.G. Cicero, Martina Rosticci, Martino Morbini, Marcella Cagnati, Elisa Grandi, \\ Angelo Parini, Claudio Borghi
}

Medical and Surgical Sciences Department, University of Bologna, Bologna, Italy

Submitted: 25 February 2015

Accepted: 23 March 2015

Arch Med Sci 2016; 12, 3: 507-512

DOI: 10.5114 /aoms.2016.59923

Copyright (C) 2016 Termedia \& Banach

\section{Abstract}

Introduction: Polyunsaturated fatty acids (PUFAs) derived from different sources could have different lipid-lowering effects in humans. The main aim of our study was to compare the short-term triglyceride-lowering efficacy of krill oil and purified omega 3 ethyl ester PUFAs in mildly overweight hypertriglyceridemic subjects.

Material and methods: This double-blind, randomized clinical trial was carried out in 25 moderately hypertriglyceridemic subjects (TG $=150-500 \mathrm{mg} /$ dl). After a 4-week run-in, participants were allocated to treatment with similar pills containing omega 3 ethyl ester PUFAs 1000 mg twice a day vs. krill oil $500 \mathrm{mg}$ twice a day. After 4 weeks of treatment, participants were asked to observe a 4-week wash-out period, and they were then assigned to the alternative treatment for a further period of 4 weeks.

Results: Although both PUFA sources were able to improve TG plasma levels, esterified omega 3 PUFAs were more efficacious than krill oil ( $p<$ $0.05)$. Nonetheless, only krill oil treatment was able to significantly improve high-density lipoprotein cholesterol and apolipoprotein Al levels, compared to both baseline $(p<0.05)$ and end of treatment with esterified omega 3 PUFAs $(p<0.05)$ values. Both treatments were able to significantly reduce high-sensitivity C-reactive protein (hs-CRP) levels from the baseline $(p<0.05)$, but krill oil improved it more efficaciously than esterified omega 3 PUFAs $(p<0.05)$.

Conclusions: Krill oil has lipid-lowering effects comparable with those obtained through a 4-fold higher dose of purified omega 3 ethyl ester PUFAs in mildly overweight hypertriglyceridemic subjects, while more efficaciously reducing hs-CRP.

Key words: krill oil, eicosapentaenoic acid, docosahexaenoic acid, omega 3 fatty acids, hyperlipidemia, inflammation.

\section{Introduction}

Polyunsaturated essential fatty acids (PUFAs), and in particular the omega 3 PUFAs eicosapentaenoic acid (EPA) and docosahexaenoic acid $(\mathrm{DHA})$, have several metabolic and pharmacological effects potentially useful in the prevention or management of a large number of human chronic diseases [1, 2]. Given this potential role in therapy, the finding of new sources of omega 3 PUFAs is always a matter of interest [3].
Corresponding author: Arrigo F.G. Cicero MD, PhD Medical and Surgical Sciences Department Sant'Orsola-Malpighi University Hospital U.O. Medicina Interna Borghi Via Albertoni 15 40138 Bologna, Italy Phone: ++39 0516362224 Fax: ++39 516826125

E-mail: arrigo.cicero@unibo.it 
An alternative source of omega 3 PUFAs is the krill (Euphausia superba), a small crustacean that lives in the Antarctic Ocean and contains many types of long-chain PUFAs. Krill and fish oil differ in their composition: krill oil is comprised of phospholipids (constituting 30-65\%) and triglycerides, while fish oil contains only triglycerides. The primary phospholipid in krill oil is phosphatidylcholine, with $40 \%$ of the total fatty acids attached to the phosphatidylcholine (PC) being EPA and DHA [4]. This composition promotes the efficiency of absorption of fatty acids into the blood, justifying the high bioavailability of omega 3 PUFAs taken in this form [4]. Krill oil also contains various potent antioxidants, including vitamins $A$ and $E$, and astaxanthin, whose capacity of absorption of oxygen radicals proved to be 34 times higher than that of coenzyme $\mathrm{Q}_{10}$, a strong antioxidant agent, and 48 times higher than that of fish oil [5].

Beyond some preliminary evidence [6], it is not fully clear what advantages the higher bioavailability of krill oil could bring in terms of lipid-lowering efficacy, in particular when compared with purified omega 3 ethyl ester PUFAs.

In this context, the main aim of our study was to comparatively evaluate the short-term triglyceride-lowering efficacy of krill oil and purified omega 3 ethyl ester PUFAs in mildly overweight hypertriglyceridemic subjects.

\section{Material and methods}

\section{Study design}

This double-blind, randomized clinical trial was carried out in 25 moderately hypertriglyceridemic subjects, non-smokers, pharmacologically untreated, in primary prevention for cardiovascular diseases, consecutively enrolled in the ambulatory service of cardiovascular disease prevention in the Medical and Surgical Sciences Department of the University of Bologna.

Inclusion criteria were: age between 18 and 70 years, and fasting plasma triglyceride (TG) level between 150 and $500 \mathrm{mg} / \mathrm{dl}$, confirmed at least in two sequential blood samples before signing the consent form.

Exclusion criteria were:

- Personal history of cardiovascular disease or risk equivalents;

- LDL-cholesterolemia > $190 \mathrm{mg} / \mathrm{dl}$ or TG > 500 $\mathrm{mg} / \mathrm{dl}$;

- Type 2 diabetes;

- Obesity (body mass index (BMI) > $30 \mathrm{~kg} / \mathrm{m}^{2}$ );

- Actual or previous (last 3 months) use of lipidlowering drugs or nutraceuticals, or drugs affecting lipid metabolism (in particular, oral corticosteroids, immunosuppressants, full dosed thiazides and $\beta$-blockers);
- Known thyroid, liver, renal or muscle diseases;

- Previous intolerance to fish oil extracts.

Smokers were excluded from the trial in order to reduce the possible effect of smoking on high-sensitivity C-reactive protein (hs-CRP) levels.

At the enrollment visit, participants were given standard behavioral and qualitative dietary suggestions to correct unhealthy habits. Standard diet advice was given by a dietitian and/or specialist doctor. Dietitians and/or specialist doctors periodically provided instructions on dietary intake recording procedures as part of a behavior modification program, and later used the subject's food diaries for counseling. In particular, participants were instructed to follow general indications of a Mediterranean diet, avoiding excessive intake of simple sugars and sweet foods, alcohol, dairy products and red meat derived products during the study, and maintaining overall constant dietary habits. Participants were also generically encouraged to increase their physical activity by walking briskly for 20 to $30 \mathrm{~min}, 3$ to 5 times per week, or by cycling.

\section{Treatments}

After 4 weeks of diet modification and advice to increase physical activity (run-in period), at baseline participants were allocated to treatment with similar pills containing omega 3 ethyl ester PUFAs 1000 mg twice a day vs. krill oil 500 mg twice a day to be taken once a day, at evening before the meal (kindly provided by Erbozeta S.r.l., San Marino, RSM). Each pill of omega 3 ethyl ester PUFAs $1000 \mathrm{mg}$ contained at least $85 \%$ of EPA and DHA, with a EPA/DHA ratio of 0.9-1.5; each krill oil pill included EPA $75 \mathrm{mg}$ and DHA $45 \mathrm{mg}$. As it was a crossover trial, after 4 weeks of treatment, participants were asked to observe a 4-week wash-out period, and they were then assigned to the alternative treatment for a further period of 4 weeks. Clinical and laboratory data were obtained at the baseline, at the end of the first treatment period, after the wash-out, and again after the second treatment period. Randomization was done using drawing of envelopes containing randomization codes prepared by an independent statistician and specific software. The envelopes were then further mixed and distributed to the investigators, who assigned the randomization code to the enrolled subjects in a progressive way. A copy of the code was provided only to the person responsible for performing the statistical analysis.

Throughout the study, we instructed participants to take the new product's first dose on the day after they received it in a blinded box. At the same time, all unused products were retrieved for the inventory. Product compliance was assessed by counting the number of product doses returned at the time of specified clinic visits. 


\section{Assessments}

All plasma parameters were obtained after a 12hour overnight fast. Venous blood samples were drawn by a nurse in all participants between 8:00 a.m. and 9:00 a.m. Plasma used was obtained by addition of $\mathrm{Na}_{2}$ EDTA $(1 \mathrm{mg}=\mathrm{ml})$ and centrifuged at $3,000 \mathrm{~g}$ for $15 \mathrm{~min}$ at $4.0^{\circ} \mathrm{C}$. Immediately after centrifugation, plasma samples were frozen and stored at $-80^{\circ} \mathrm{C}$ for no more than 3 months. The following parameters were evaluated via standardized methods [7]: total cholesterol (TC), high-density lipoprotein cholesterol (HDL-C), TG, non HDL-C, apolipoprotein B (apoB), apolipoprotein Al (apoAI), glucose, creatinine, liver transaminases and creatinine phosphokinase (CPK). All measurements were centrally performed in the laboratory of our department. LDL cholesterol (LDL-C) was estimated with the Friedewald formula.

High-sensitivity C-reactive protein (hs-CRP) was also measured using latex-enhanced immunonephelometric assays on a BN II analyzer (Dade Behring, Newark, Delaware, USA). The intra-assay and inter-assay coefficients of variation (CVs) were 5.7 and $1.3 \%$, respectively [8].

\section{Statistical analysis}

Data were analyzed using intention to treat by means of the Statistical Package for the Social Sciences (SPSS) 19.0, version for Windows. Normally distributed baseline characteristics of the population were compared using Student's $t$-test and $\chi^{2}$ test followed by Fisher's exact test for categorical variables. Two-way analyses of variance for cross-over design were used to assess the effect of treatment during the consumption of esterified omega 3 PUFAs and krill oil. All data are expressed as means and SD. To verify the basic assumptions of cross-over design, besides the evaluation of the period effect, the presence of a carryover effect was excluded. A $p$ level of $<0.05$ was considered significant for all tests.

\section{Results}

At the baseline, the enrolled participants had similar BMI and lipid pattern, and were age and sex matched. The baseline characteristics of participants assigned to the two different types of treatment (esterified omega 3 PUFAs-krill oil or krill oil-esterified omega 3 PUFAs) were similar, and no significant differences were observed regarding the parameters studied (Table I). No participant dropped out from the study because of adverse events, and compliance with the treatment was almost total in both groups and treatment periods.

From the randomization visit to the end of the study, the enrolled subjects maintained overall a similar dietary pattern, without a significant change in total energy, total cholesterol and total saturated fatty acid intake.

The main results of the trial are shown in Table II.

Comparing the effects of the two tested treatments, both PUFA sources were able to lower TG plasma levels, but esterified omega 3 PUFAs were more efficacious than krill oil $(p<0.05)$. In contrast, only krill oil treatment was able to significantly improve HDL-C and apoAl level, both compared to baseline $(p<0.05)$ and end of treatment with esterified omega 3 PUFAs $(p<0.05)$ values. Compared to the baseline values, non-HDL cholesterol improved only after krill oil treatment $(p<0.05)$, and not after treatment with esterified omega 3 PUFAs. Finally, both treatments were able to significantly reduce hs-CRP levels from the baseline $(p<0.05)$, but krill oil improved hs-CRP more efficaciously than esterified omega 3 PUFAs $(p<0.05)$.

No significant difference was detected comparing pre- and post-treatment values as regards TC, LDL-C, ApoB, fasting plasma glucose (FPG), creatinine, liver transaminases and CPK in each of the treatment groups.

\section{Discussion}

The therapeutic value of omega 3 PUFAs, mainly (but not only) found in fish oils, EPA and DHA, has been extensively studied in a wide variety of disease conditions, first of all in cardiovascular disease. In fact, PUFAs have a large number of pharmacological properties that justify their dietary supplementation in a considerable number of human diseases $[9,10]$.

The significant difference in efficacy observed in various conditions depends of course on the wide variation in baseline omega 3 PUFAs status in study participants (often even not tested) [11], the use of inappropriate dosage and the possible wrong ratio between EPA and DHA. On the other hand, it seems that the difference in efficacy with equal dosages could be at least partly be related to the large discrepancy in quality of the product and to the bioavailability of the omega 3 PUFAs. In particular, the contrasting results observed with supplementation of omega 3 PUFAs in cardiovascular disease prevention or treatment have been attributed to the fact that the serum level of omega 3 PUFAs is more strongly linked to the clinical outcome than to the omega 3 PUFA dose used $[12,13]$. In this context, testing the different metabolic effects of omega 3 PUFAs with different bioavailability could be of relevant interest [3].

In our study, we observed that $1000 \mathrm{mg} /$ day of krill oil has lipid-lowering effects comparable with those achieved through a higher dose of purified omega 3 ethyl ester PUFAs in mildly overweight 
Table I. Main characteristics of the investigated parameters at baseline in patients treated with the tested krill oil or esterified omega 3 PUFAs

\begin{tabular}{|c|c|c|c|c|}
\hline \multirow[t]{2}{*}{ Parameter } & \multicolumn{2}{|c|}{$\begin{array}{l}\text { Esterified omega } 3 \text { PUFAS } \\
\quad(n=12 ; M=7, F=6)\end{array}$} & \multicolumn{2}{|c|}{$\begin{array}{c}\text { Krill oil } \\
(n=13 ; M=7, F=6)\end{array}$} \\
\hline & Mean & SD & Mean & SD \\
\hline Age [years] & 50.3 & 5.6 & 51.1 & 6.5 \\
\hline Total energy intake [kcal] & 2264 & 131 & 2318 & 149 \\
\hline Fat dietary intake (\% of total energy) & 27.8 & 3.0 & 27.4 & 2.9 \\
\hline $\begin{array}{l}\text { Saturated fat intake (fat dietary intake } \\
\text { (\% on total energy)) }\end{array}$ & 9.1 & 1.1 & 9.2 & 0.9 \\
\hline Protein intake (\% of total energy) & 17.7 & 2.1 & 18.3 & 1.9 \\
\hline Carbohydrate intake (\% of total energy) & 51.3 & 4.2 & 52.1 & 4.4 \\
\hline Cholesterol intake [mg] & 179.1 & 9.2 & 177.8 & 9.8 \\
\hline $\mathrm{BMI}\left[\mathrm{kg} / \mathrm{m}^{2}\right]$ & 27.6 & 1.7 & 27.9 & 1.2 \\
\hline $\mathrm{SBP}[\mathrm{mm} \mathrm{Hg}]$ & 135.1 & 5.7 & 135.0 & 5.5 \\
\hline $\mathrm{DBP}[\mathrm{mm} \mathrm{Hg}]$ & 85.6 & 4.0 & 83.3 & 5.5 \\
\hline $\mathrm{PP}[\mathrm{mm} \mathrm{Hg}]$ & 49.4 & 4.7 & 51.6 & 5.2 \\
\hline $\mathrm{MAP}[\mathrm{mm} \mathrm{Hg}]$ & 110.4 & 4.3 & 109.2 & 4.9 \\
\hline $\mathrm{TC}[\mathrm{mg} / \mathrm{dl}]$ & 194.6 & 14.1 & 187.0 & 12.8 \\
\hline $\mathrm{HDL}-\mathrm{C}[\mathrm{mg} / \mathrm{dl}]$ & 39.5 & 6.7 & 39.8 & 8.1 \\
\hline LDL-C [mg/dl] & 94.2 & 15.4 & 97.1 & 13.8 \\
\hline TG [mg/dl] & 369.2 & 26.8 & 362.4 & 27.7 \\
\hline Non-HDL-C [mg/dl] & 165.3 & 26.5 & 161.2 & 34.5 \\
\hline ApoAl [mg/dl] & 115.5 & 17.2 & 120.4 & 17.9 \\
\hline ApoB $[\mathrm{mg} / \mathrm{dl}]$ & 111.1 & 12.3 & 114.0 & 13.5 \\
\hline FPG [mg/dl] & 92.1 & 7.7 & 95.3 & 9.38 \\
\hline Creatinine $[\mathrm{mg} / \mathrm{dl}]$ & 1.1 & 0.1 & 1.05 & 0.13 \\
\hline CPK $[U / I]$ & 121.6 & 37.0 & 131.6 & 26.9 \\
\hline AST [U/I] & 21.3 & 4.9 & 21.2 & 4.8 \\
\hline ALT [U/I] & 24.5 & 5.1 & 23.6 & 5.4 \\
\hline hs-CRP [mg/l] & 1.97 & 0.35 & 2.15 & 0.43 \\
\hline
\end{tabular}

$B M I$ - body mass index, SBP - systolic blood pressure, DBP - diastolic blood pressure, PP - pulse pressure, MAP - mean arterial pressure, TC - total cholesterol, LDL-C - low-density lipoprotein cholesterol, HDL-C - high-density lipoprotein cholesterol, TG - triglycerides, Apo apolipoprotein, FPG - fasting plasma glucose, CPK - creatine-phosphokinase, AST - aspartate transaminase, ALT - alanine transaminase, hs-CRP-high-sensitivity C-reactive protein. Descriptive values reported as mean and standard deviation. The subjects were cross-matched for all the tested variables.

hypertriglyceridemic subjects, being slightly less efficacious in terms of triglyceride reduction, but significantly improving the HDL cholesterol level. These data are partly in agreement with those already known from previous studies [6, 14], even if the results are hardly comparable because of the use of different dosages and omega 3 formulations.

Even if the marine omega 3 PUFAs are known to exert a significant anti-inflammatory effect [15], the higher reduction in hs-CRP observed during krill oil treatment is also compatible with the anti-inflammatory effects previously observed in another short-term clinical trial where similar krill oil dosages were tested [16]. The anti-inflammatory effect of krill oil could be due to its direct antioxidant action, but also to its modulating effect on endocannabinoid metabolism [17].

Moreover, the formulation we tested at the doses prescribed have similar cost per day of therapy. 
Table II. Changes in the investigated parameters after esterified omega 3 PUFAs and krill oil treatment (cumulative data comparing pre-/post-treatment phases; ${ }^{*} p<0.05$ vs. esterified omega 3 PUFAs)

\begin{tabular}{|c|c|c|c|c|c|c|}
\hline \multirow[t]{2}{*}{$\Delta(\mathrm{T} 1-\mathrm{T0})$} & \multicolumn{3}{|c|}{$\begin{array}{l}\text { Esterified omega } 3 \\
\text { PUFAS }(n=25)\end{array}$} & \multicolumn{3}{|c|}{ Krill oil $(n=25)$} \\
\hline & Mean & SD & $P$ vs. baseline & Mean & SD & $P$ vs. baseline \\
\hline$\Delta \mathrm{TC}[\mathrm{mg} / \mathrm{dl}]$ & -2.1 & 3.4 & 0.397 & -3.8 & 4.1 & 0.078 \\
\hline$\Delta \mathrm{HDL}-\mathrm{C}[\mathrm{mg} / \mathrm{dl}]$ & +1.4 & 4.9 & 0.156 & $+4.5^{*}$ & 2.1 & 0.039 \\
\hline$\Delta \mathrm{LDL}-\mathrm{C}[\mathrm{mg} / \mathrm{dl}]$ & -1.9 & 3.8 & 0.462 & -3.7 & 3.9 & 0.111 \\
\hline$\Delta \mathrm{TG}[\mathrm{mg} / \mathrm{dl}]$ & -72.8 & 28.9 & 0.001 & $-55.7^{*}$ & 18.3 & 0.003 \\
\hline$\Delta$ Non-HDL-C $[\mathrm{mg} / \mathrm{dl}]$ & -3.1 & 3.8 & 0.069 & $-8.1^{*}$ & 4.7 & 0.009 \\
\hline$\Delta$ ApoAl $[\mathrm{mg} / \mathrm{dl}]$ & +11.1 & 20.9 & 0.060 & +19.4 & 15.1 & 0.049 \\
\hline$\Delta \mathrm{ApoB}[\mathrm{mg} / \mathrm{dl}]$ & -2.8 & 8.8 & 0.958 & -2.6 & 5.45 & 0.529 \\
\hline$\Delta \mathrm{FPG}[\mathrm{mg} / \mathrm{dl}]$ & +1.9 & 8.5 & 0.665 & +2.2 & 11.4 & 0.758 \\
\hline$\Delta$ Creatinine $[\mathrm{mg} / \mathrm{dl}]$ & -0.07 & 0.21 & 0.087 & -0.03 & 0.14 & 0.317 \\
\hline$\Delta \mathrm{CPK}[\mathrm{U} / \mathrm{l}]$ & +6.8 & 34.35 & 0.569 & +4.4 & 44.3 & 0.668 \\
\hline$\Delta \mathrm{AST}[\mathrm{U} / \mathrm{l}]$ & +4.5 & 11.7 & 0.363 & +2.2 & 4.8 & 0.133 \\
\hline$\Delta \mathrm{ALT}[\mathrm{U} / \mathrm{l}]$ & +5.4 & 14.7 & 0.360 & +1.1 & 10.0 & 0.581 \\
\hline$\Delta \mathrm{hs}-\mathrm{CRP}[\mathrm{mg} / \mathrm{l}]$ & -0.14 & 0.07 & 0.030 & $-0.35^{*}$ & 0.12 & $<0.001$ \\
\hline
\end{tabular}

TC - total cholesterol, LDL-C - low-density lipoprotein cholesterol, HDL-C - high-density lipoprotein cholesterol, TG - triglycerides, Apo apolipoprotein, FPG - fasting plasma glucose, CPK - creatine-phosphokinase, AST - aspartate transaminase, ALT - alanine transaminase, hs-CRP - high-sensitivity C-reactive protein. A two-way analysis of variance for crossover design was used to assess the effects of treatment during treatment with esterified omega 3 PUFAs or krill oil ( ${ }^{*} p<0.05$ vs. esterified omega 3 PUFAs). All the data are expressed as mean and SD.

Our observations, combined with those of other authors showing positive effects of krill oil on cognitive functions [18] and on prevention of fatty acid accumulation in the liver [19], support the interest in extending the knowledge of the possible usefulness of this natural product in the management of cardiovascular disease risk [20]. Overall, our data suggest the need to intensify research on the pleiotropic effects of krill oil, especially in association with other preventive drugs such as antidiabetics or statins [21,22].

Of course, our pilot study has some relevant limitations. The first is the small patient sample, even though the study strength is improved by a crossover design where participants are all treated with both placebo and active treatment. Another limitation is the short duration of the study. A longer trial would not have been considered ethical as a preliminary report, considering that the use of ethyl-ester omega 3 fatty acids in the participants enrolled in our trial is supported by a large and consistent literature. Also, we did not dose the plasma level of EPA and DHA, deducing our conclusion from the observed changes in hematochemical parameters. Finally, efficacy data were based on a relatively low number of laboratory parameters, and not on instrumental ones (as for instance flow-mediated vasodilation or pulse wave velocity). However, the study duration was too short to observe any anatomical change in vessel structures, while the considered laboratory parameters are validated biomarkers of vascular stress.

In conclusion, based on our data, a nutraceutical containing krill oil 1000 mg/day has lipid-lowering effects comparable with those achieved through a higher dose of purified omega 3 ethyl ester PUFAs in mildly overweight hypertriglyceridemic subjects, being slightly less efficacious in terms of triglyceride reduction but significantly improving the HDL cholesterol level and more efficaciously reducing hs-CRP. These results have to be confirmed in larger patient samples and longer studies.

Further trials are needed to evaluate the longterm efficacy and tolerability profile of krill oil in frail patients, such as hypercholesterolemic elderly [23] and statin-intolerant ones [24].

\section{Acknowledgments}

This trial has been approved by the local ethics committee and has therefore been performed in accordance with the ethical standards laid down in the 1964 Declaration of Helsinki and its later amendments. All persons involved in the trial signed an informed consent form prior to their inclusion in the study. 


\section{Conflict of interest}

The authors declare no conflict of interest.

\section{References}

1. Cicero AF, De Sando V, Parini A, Borghi C. Polyunsaturated fatty acids application in internal medicine: beyond the established cardiovascular effects. Arch Med Sci 2012; 8: 784-93.

2. Cicero AF, Ertek S, Borghi C. Omega-3 polyunsaturated fatty acids: their potential role in blood pressure prevention and management. Curr Vasc Pharmacol 2009; 7: 330-7.

3. Cicero AF, Morbini M, Borghi C. Do we need "new" omega 3 polyunsaturated fatty acids formulations? Exp Opin Pharmacother 2014; 4: 1-4.

4. Ramprasath VR, Eyal I, Zchut S, Jones PJH. Enhanced increase of omega 3 index in healthy individuals with response to 4 week n-3 fatty acid supplementation from krill oil versus fish oil. Lipids Health Dis 2013; 12: 178.

5. Naguib YM. Antioxidant activities of astaxanthin and related carotenoids. J Agric Food Chem 2000; 48: 1150-4.

6. Ulven SM, Kirkhus B, Lamglait A, et al. Metabolic effects of krill oil are essentially similar to those of fish oil but at lower dose of EPA and DHA, in healthy volunteers. Lipids 2011; 46: 37-46.

7. Cicero AF, Derosa G, Parini A, et al. Red yeast rice improves lipid pattern, high-sensitivity C-reactive protein, and vascular remodeling parameters in moderately hypercholesterolemic Italian subjects. Nutr Res 2013; 33: 622-8.

8. Rifai N, Tracy RP, Ridker PM. Clinical efficacy of an automated high-sensitivity C-reactive protein assay. Clin Chem 1999; 45: 2136-41.

9. Derosa G, Limas CP, Macías PC, Estrella A, Maffioli P. Dietary and nutraceutical approach to type 2 diabetes. Arch Med Sci 2014; 10: 336-44.

10. Mariscalco G, Sarzi Braga S, Banach M, et al. Preoperative $n-3$ polyunsatured fatty acids are associated with a decrease in the incidence of early atrial fibrillation following cardiac surgery. Angiology 2010; 61: 643-50.

11. Banach M, Serban C, Aronow WS, et al. Lipid, blood pressure and kidney update 2013. Int Urol Nephrol 2014; 46: 947-61.

12. Superko HR, Superko AR, Lundberg GP, et al. Omega-3 fatty acid blood levels clinical significance update. Curr Cardiovasc Risk Rep 2014; 8: 407.

13. Ghasemifard S, Turchini GM, Sinclair AJ. Omega-3 long chain fatty acid "bioavailability": a review of evidence and methodological considerations. Prog Lipid Res 2014; 56C: 92-108.

14. Bunea R, El Farrah K, Deutsch L. Evaluation of the effects of neptune krill oil on the clinical course of hyperlipidemia. Alt Med Rev 2004; 9: 420-8.

15. Calder PC. Marine omega-3 fatty acids and inflammatory processes: effects, mechanisms and clinical relevance. Biochim Biophys Acta 2015; 1851: 469-84.

16. Deutsch L. Evaluation of the effect of Neptune Krill Oil on chronic inflammation and arthritic symptoms. J Am Coll Nutr 2007; 26: 39-48.

17. Banni S, Carta G, Murru E, et al. Krill oil significantly decreases 2-arachidonoylglycerol plasma levels in obese subjects. Nutr Metab 2011; 8: 7.

18. Konagai C, Yanagimoto K, Hayamizu K, Han L, Tsuji T, Koga Y. Effects of krill oil containing n-3 polyunsaturated fatty acids in phospholipid form on human brain function: a randomized controlled trial in healthy elderly volunteers. Clin Interv Aging 2013; 8: 1247-57.

19. Ferramosca A, Zara V. Modulation of hepatic steatosis by dietary fatty acids. World J Gastroenterol 2014; 20: 1746-55.

20. Cicero AF, Colletti A. Krill oil: evidence of a new source of polyunsaturated fatty acids with high bioavailability. Clin Lipidol 2015; 10: 1-4.

21. Sikora J, Kostka B, Marczyk I, Krajewska U, Chałubiński $M$, Broncel $M$. Effect of statins on platelet function in patients with hyperlipidemia. Arch Med Sci 2013; 9: 622-8.

22. Guardado-Mendoza R, Prioletta A, Jiménez-Ceja LM, Sosale A, Folli $F$. The role of nateglinide and repaglinide, derivatives of meglitinide, in the treatment of type 2 diabetes mellitus. Arch Med Sci 2013; 9: 936-43.

23. Aronow WS. Lipid-lowering therapy in older persons. Arch Med Sci 2015; 11: 43-56.

24. Banach M, Rizzo M, Toth PP, et al. Statin intolerance an attempt at a unified definition. Position paper from an International Lipid Expert Panel. Arch Med Sci 2015; 11: 1-23. 